\title{
Política monetaria en el modelo con expectativas, curva de Phillips: práctica de ordenador
}

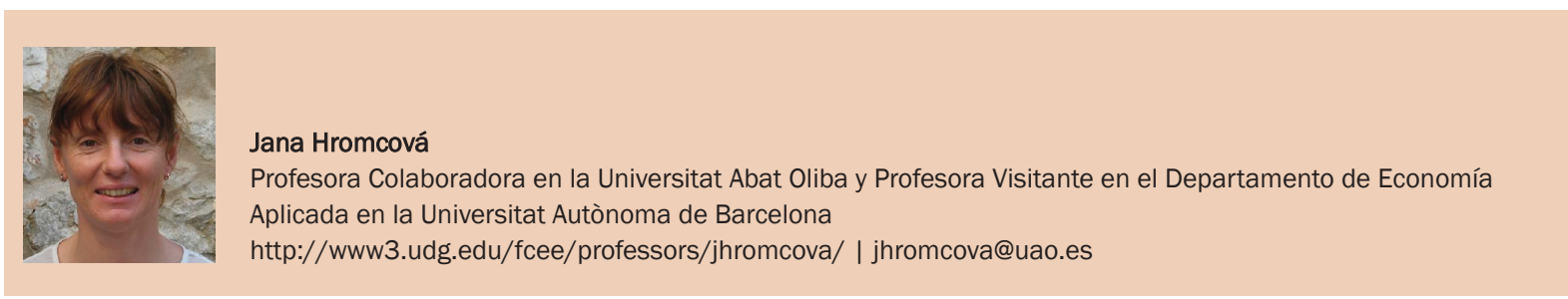

|Fecha presentación: 05/10/2009 | Aceptación: 06/11/2009 |Publicación: 04/12/2009

\section{Resumen}

Recomendamos un ejercicio numérico que permite a los estudiantes observar la dinámica de un simple modelo, compuesto por la curva de Phillips, ley de Okun y demanda agregada, ante variaciones en la política monetaria. El ejercicio tiene como objetivo enseñar a los estudiantes la importancia de la manera en la que se forman las expectativas y la (no) credibilidad del banco central para el cumplimiento de los objetivos de la política monetaria.

Palabras clave: política monetaria, curva de Phillips, expectativas adaptativas, expectativas racionales, credibilidad del banco central

\section{Resum}

Recomanem un exercici numèric que permet als estudiants observar la dinàmica d'un simple model, compost per la corba de Phillips, llei d'Okun i demanda agregada, davant variacions en la política monetària. L'exercici té com a objectiu mostrar als estudiants la importància de la manera en què es formen les expectatives i la (no) credibilitat del banc central per a aconseguir els objectius de la política monetària.

Paraules clau: política monetària, corba de Phillips, expectatives adaptatives, expectatives racionals, credibilitat del banc central

\footnotetext{
Abstract

We recommend a numerical exercise that allows students to observe the dynamics of a simple model (which consists of the Phillips curve, Okun's law and aggregate demand) under variations in the monetary policy. The objective of the proposed exercise is to show the students the importance of the way the expectations are formed and the central bank (non) credibility in the accomplishment of the monetary policy goals.
}

Keywords: monetary policy, Phillips curve, adaptative expectations, rational expectations, central bank credibility 


\section{Introducción}

En este trabajo recomendamos un ejercicio numérico que permite a los estudiantes observar la dinámica de un simple modelo, compuesto por la curva de Phillips, la ley de Okun y la demanda agregada, ante variaciones en la política monetaria.

El tema tratado está diseñado para su uso en la asignatura Macroeconomía avanzada en la licenciatura de Economía, pero también se puede adaptar a la Macroeconomía intermedia en la licenciatura de Administración y Dirección de Empresas y/o Economía. El ejercicio corresponde en parte al tema 9 de Blanchard (2006) y el Tema 3 de Novales y Sebastián (1999).

El objetivo de esta actividad es profundizar en el conocimiento del comportamiento de la economía en el contexto de expectativas adaptativas y expectativas racionales. El alumno debe hacer decisiones sobre la ejecución de la política monetaria según los objetivos establecidos. Si la economía se enfrenta a una variación en la política monetaria (anunciada), la simulación numérica del modelo permite ver lo siguiente: i) cuando asumimos que los agentes forman sus expectativas sobre la inflación del periodo siguiente de forma adaptativa, observamos ciclos durante la transición de la economía hacia el nuevo equilibrio; ii) cuando asumimos que los agentes forman sus expectativas sobre la inflación del periodo siguiente de forma racional, la economía se traslada directamente al nuevo equilibrio, evitando la transición, siempre y cuando el banco central sea creíble.

Por un lado, la práctica permite comprobar que una variación progresiva de la oferta monetaria puede conseguir el objetivo de la desinflación a menor coste, en términos del desempleo, que una variación súbita. Por otro lado, se puede observar que la credibilidad del banco central es un factor muy importante que puede ayudar a conseguir los objetivos de la autoridad monetaria de manera mucho más eficaz. La credibilidad del banco central sólo se puede garantizar si la institución monetaria no depende del gobierno y su actuación no está motivada por fines electorales.

Las competencias que se trabajan a lo largo de esta actividad son las siguientes: analizar efectos de políticas económicas, identificar causas de las fluctuaciones, formular los problemas económicos a través de modelos matemáticos, aplicar herramientas informáticas para resolver modelos teóricos, y redactar un informe.

El resto del artículo desarrolla de la manera siguiente: en la sección 2 especificamos el modelo, en la sección 3 comentamos los resultados, y concluimos en la sección 4.

\section{Modelo}

Compondremos el modelo de tres elementos: la ley de Okun, la curva de Phillips y la demanda agregada.

La ley de Okun es una relación empírica entre la variación de la tasa de paro y el crecimiento del output

$$
u_{t}-u_{t-1}=-\alpha\left(\gamma_{Y t}-\bar{\gamma}_{Y_{N}}\right)
$$

donde $u_{t}$ es la tasa de paro en el período $t$ (por tanto $u_{t-1}$ es la tasa de paro en el período $t-1$ ), $\alpha$ es una constante positiva, $\gamma_{Y_{t}}$ es la tasa de crecimiento del output real entre $t$ y $t-1$ y $\bar{\gamma}_{Y_{N}}$ es la tasa normal de crecimiento del output real. La tasa normal de crecimiento del output representa el equilibrio. La relación (1) implica que para que no aumente la tasa de desempleo, el output real debe crecer al menos a una

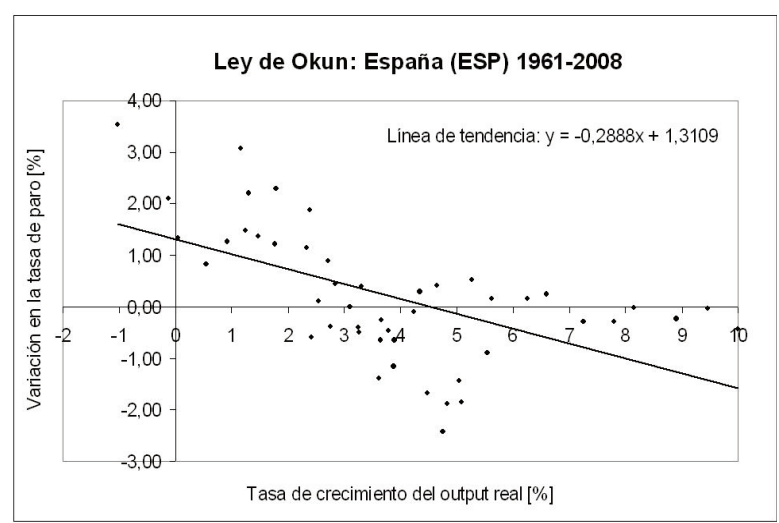

Figura 1: La ley de Okun, España, datos anuales, periodo 1961-2008. Fuente de datos: OCDE Main Economic Indicators (2009).

tasa $\bar{\gamma}_{Y_{N}}$. En la Figura 1 ilustramos la ley de Okun con los datos españoles anuales para el período 1961-2008. Vemos que la tasa normal de crecimiento para este periodo es aproximadamente del $4,5 \%$. La pendiente de la línea de regresión corresponde al valor de $\alpha$. El parámetro a mide el efecto que produce un crecimiento de la producción mayor de lo normal en la variación de la tasa de desempleo. En el caso estudiado (España entre 1961 y 2008), un aumento de la tasa de crecimiento de $1 \%$ mayor de lo normal durante un año provoca una reducción en la tasa de paro de $0,3 \%$.

El siguiente elemento del modelo es la demanda agregada, una relación entre el precio y el nivel de output que se ve afectada por las políticas económicas

$$
Y_{t}=Y\left(\frac{M_{t}}{P_{t}}, G_{t}, T_{t}\right)
$$

donde $Y_{t}$ es el output real en $t, M_{t}$ es la oferta monetaria nominal en $t, P_{t}$ es el nivel de precios en $t, G_{t}$ es el gasto público en $t$ y $T_{t}$ es el nivel de impuestos en $t$. Como en nuestro caso nos interesa estudiar los efectos de la política monetaria, asumimos que la política fiscal no afecta a la demanda agregada, $G_{t}=0$ y $T_{t}=0$ para todos los periodos del tiempo,

$$
Y_{t}=Y\left(\frac{M_{t}}{P_{t}}\right) \text {. }
$$

Esta relación podemos escribir, por ejemplo, como

$$
Y_{t}=\xi \frac{M_{t}}{P_{t}}
$$

donde $\xi$ es una constante positiva. Escribiendo la ecuación (4) en tiempo continuo, tomando logaritmos de los dos lados y la derivada con respecto al tiempo obtenemos

$$
\gamma_{Y t}=\gamma_{M t}-\pi_{t}
$$

donde

$$
\gamma_{Y t}=\frac{Y_{t}-Y_{t-1}}{Y_{t-1}}
$$

es la tasa de crecimiento del output real entre el periodo ty $t-1$

$$
\gamma_{M t}=\frac{M_{t}-M_{t-1}}{M_{t-1}}
$$

es la tasa de crecimiento de la oferta monetaria nominal entre el periodo $t \mathrm{y} t-1 \mathrm{y}$ 


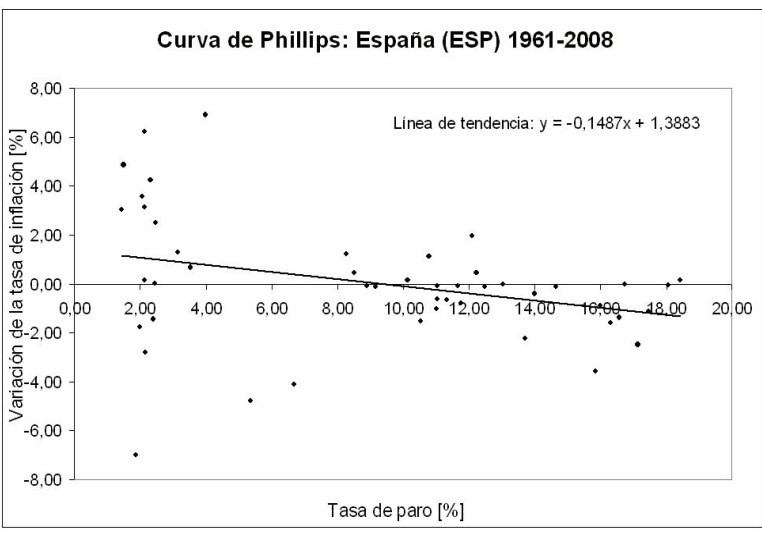

Figura 2: La curva de Phillips, España, datos anuales, periodo 1961-2008. Fuente de datos: OCDE Main Economic Indicators (2009).

$$
\pi_{t}=\frac{P_{t}-P_{t-1}}{P_{t-1}}
$$

es la tasa de inflación entre el periodo $t$ y $t$-1. De esta manera convertimos la relación entre el output, la oferta monetaria y el precio a una relación entre sus respectivas tasas de crecimiento, ecuación (5).

La última ecuación de nuestro modelo es la relación entre la variación de la tasa de inflación y la tasa de paro, la curva de Phillips aumentada con expectativas ${ }^{1}$. La curva de Phillips tiene la siguiente forma analítica

$$
\pi_{t}-\pi_{t}^{e}=-\beta\left(u_{t}-u_{N}\right)
$$

donde $\pi_{t}$ es la tasa de inflación en $t$ y $\pi_{t}^{e}$ es la inflación esperada en $t-1$ para $t, \beta$ es una constate positiva, $u_{t}$ es la tasa de paro en el período $t$ y $u_{N}$ es la tasa de paro natural. La tasa de paro natural es también conocida como NAIRU - NonAccelerating Inflation Rate of Unemployment - literalmente, tasa de desempleo no aceleradora de la inflación.

La elección de la oferta monetaria estará en manos de la autoridad monetaria. Dada la tasa de crecimiento de la oferta nominal del dinero, las otras variables se ajustarán para que se cumplan todas las relaciones del modelo. El objetivo del ejercicio es ver que el proceso de formación de expectativas puede afectar a la evolución de la economía.

2. 1 Expectativas

El elemento crucial del modelo es la especificación de las expectativas. Vamos a efectuar dos ejercicios diferentes, dependiendo del supuesto sobre la formación de expectativas: i) los agentes basarán sus expectativas sobre el futuro observando el pasado mirando solamente al pasado; ii) los agentes basarán sus expectativas sobre el futuro utilizando eficientemente toda la información del pasado, pero mirarán siempre hacia el futuro.

2.1.1 Expectativas adaptativas

$\mathrm{Si}$ asumimos que las expectativas se forman de manera totalmente adaptativa, es decir, que la expectativa que hacemos ayer sobre la inflación de hoy es la tasa de inflación que observamos ayer,

$$
\pi_{t}^{e}=\pi_{t-1}
$$

podemos escribir la curva de Phillips

$$
\pi_{t}-\pi_{t-1}=-\beta\left(u_{t}-u_{N}\right) .
$$

En la Figura 2 dibujamos la curva de Phillips usando los datos españoles anuales para el período 1961-2008. Vemos que la tasa natural de paro es muy alta, de un $10 \%$ y $\beta \cong 1 / 7$. Este valor de $\beta$ implica que, una tasa de desempleo un $1 \%$ superior a la natural durante un año, provoca una reducción de la tasa de inflación de unos $0,14 \%$.

2.2 Expectativas racionales

Si modificamos las expectativas podemos obtener un modelo con precios flexibles. Asumimos que las expectativas se forman de manera racional. Si para la expectativa que hacemos ayer sobre la inflación de hoy utilizamos eficientemente toda la información disponible hasta ayer, trataremos con expectativas racionales

$$
\pi_{t}^{e}=E_{t-1}\left(\pi_{t} ; I_{t-1}\right)
$$

donde $E_{t}$ es la esperanza matemática e $I_{t}$ es el set de información disponible hoy. Podemos escribir la curva de Phillips de la manera siguiente

$$
\pi_{t}-E_{t-1}\left(\pi_{t}\right)=-\beta\left(u_{t}-u_{N}\right) .
$$

La expectativa sobre la tasa de inflación dependerá de la política monetaria anunciada y la credibilidad del banco central.

2.3 Equilibrio

En este punto hemos especificado dos modelos diferentes. Podemos analizar el efecto de la política monetaria sobre la tasa de crecimiento, la tasa de paro y la tasa de inflación: i) para las expectativas adaptativas del sistema de ecuaciones (1), (5) y (8); y ii) para las expectativas racionales del sistema de ecuaciones (1), (5) y (10).

Para un punto inicial $\pi_{O}$ y $u_{O}$, la secuencia de la oferta monetaria $\left\{\gamma_{M}\right\}_{t=0}^{\infty}$ y la secuencia de la tasa de inflación esperada $\left\{\pi_{t}^{e}\right\}_{t=1}^{\infty}$, el sistema de ecuaciones (1), (5) y (8) o (1), (5) y (10) tiene una solución única: la trayectoria de las tasas de inflación, las tasas de paro y las tasas de crecimiento, $\left\{\pi_{t+1}, u_{t+1}, \gamma_{Y_{t}}\right\}_{t=0}^{\infty}$.

Si la oferta monetaria se mantiene constante:

$$
\gamma_{M t}=\gamma_{M t-1}=\gamma_{M}
$$

a largo plazo la economía llegará al estado estacionario. El estado estacionario está caracterizado por las siguientes condiciones:

$$
\begin{aligned}
& u_{t}=u_{t-1}=\bar{u}=u_{N}, \\
& \gamma_{Y_{t}}=\gamma_{Y_{t-1}}=\bar{\gamma}_{Y_{N}}, \\
& \pi_{t}=\pi_{t}^{e}=\bar{\pi}=\gamma_{M}
\end{aligned}
$$

donde las variables con barra $\bar{u}, \bar{\gamma}_{Y_{x}}$ y $\bar{\pi}$ son los valores estacionarios que alcanzan la tasa de paro, la tasa de crecimiento y la tasa de inflación.

Considerando el modelo con expectativas adaptativas, descrito por el sistema de ecuaciones (1), (5) y (8), la econo-

${ }^{1}$ La curva de Phillips original es una relación entre la tasa de inflación y la tasa de paro. Phelps sugirió incluir expectativas a la curva de Phillips original, dado que en un entorno de inflación positiva no cabe esperar que la inflación sea cero. La curva de Phelps se llama la curva de Phillips aumentada con expectativas, o simplemente la curva de Phillips. A lo largo de este artículo nos referiremos a la curva de Phillips aumentada con expectativas como la curva de Phillips. 


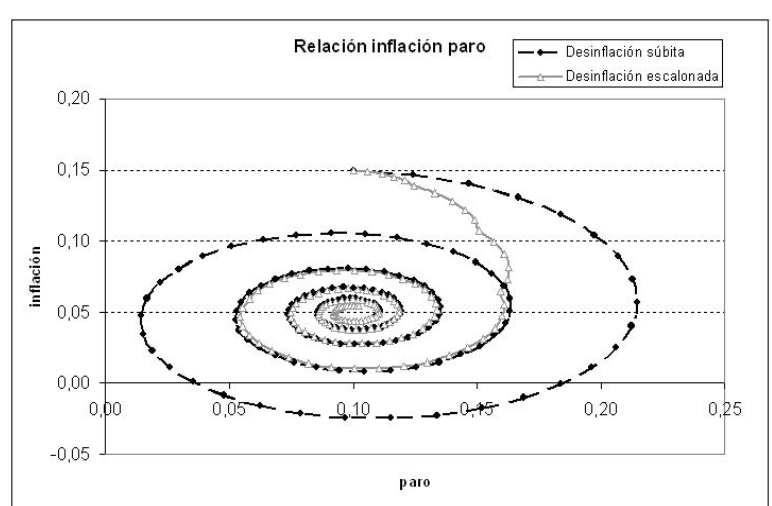

Figura 3: El diagrama de fase para el caso de expectativas adaptativas: (i) desinflación súbita; (ii) desinflación escalonada. Fuente: cálculos del autor; solución al sistema de ecuaciones (1), (5) y (8).

mía experimenta una fase de transición siempre y cuando las expectativas no sean correctas. A lo largo de la transición las tasas de inflación se van ajustando, la tasa de inflación esperada está cada vez más cerca de la actual, y la economía converge a su estado estacionario. Los precios en este caso se comportan como rígidos, y el modelo tiene características de una economía keynesiana. Si calculamos la evolución de todas las variables a lo largo del tiempo bajo el supuesto de las expectativas adaptativas, obtenemos oscilaciones en la tasa de inflación, la tasa de paro y la tasa de crecimiento. De acuerdo con las conclusiones, por ejemplo del modelo IS-LM, un(a) aumento (reducción) de la tasa de crecimiento de los saldos nominales provocará una expansión (recesión), y un(a) aumento (reducción) de la tasa de inflación a corto plazo.

En el caso del modelo con expectativas racionales, el sistema (1), (5) y (10), si las expectativas son correctas, la economía se encuentra en equilibrio. Normalmente es así, siempre y cuando no haya incertidumbre en el modelo y el banco central es creíble. Una variación en las expectativas implica la misma variación en la tasa de inflación. Eso significa que los precios son flexibles y tratamos con un modelo clásico. Si las expectativas son incorrectas, las variables se sitúan en valores distintos a los que corresponden al equilibrio, y puede haber transición. Esto puede ocurrir en el caso de que el banco central no sea creíble o que la economía se vea afectada por unos shocks aleatorios.

\section{Resultados}

El ejercicio que planteamos está detallado en el Anexo. El objetivo del ejercicio es efectuar una desinflación. Se trata de ver la evolución de la economía ante una variación de la política monetaria que permite disminuir la tasa de inflación del 15\% al 5\%. Encontramos la solución numérica utilizando el programa Excel. Los alumnos deben empezar a elaborar la hoja de Excel fijando una política monetaria, los valores iniciales de la tasa de inflación y la tasa de paro. Luego utilizando el sistema de ecuaciones (1), (5) y (8) y (1), (5) y (10), respectivamente, pueden encontrar los valores de la tasa de crecimiento de output $\gamma_{Y t}$, la tasa de paro del periodo siguiente $u_{t+1} \mathrm{y}$ la tasa de inflación del periodo siguiente $\pi_{t+1}$.

Nuestra economía tiene la tasa de paro natural del 10\% y la tasa de crecimiento normal del 4,5\%. La Figura 3 re- sume el comportamiento de la tasa de inflación y la tasa de paro a lo largo de la transición en el caso de las expectativas totalmente adaptativas. Comparamos dos casos: (i) una desinflación del $15 \%$ al $5 \%$ súbita (se baja de golpe el crecimiento de la oferta monetaria); y (ii) una desinflación del $15 \%$ al $5 \%$ escalonada (se va disminuyendo la tasa de crecimiento de la oferta monetaria poco a poco, hasta el nivel que permite alcanzar la inflación deseada). Vemos cómo la desinflación escalonada no daña tanto a la economía (la tasa de paro se mantiene en niveles más bajos y, consecuentemente, la tasa de crecimiento de la economía será más alta) y por esa razón es más recomendable. Los puntos y triángulos en la Figura 3 se repiten con una frecuencia de un periodo en el modelo.

En el caso de expectativas racionales, si el banco central es creíble, una vez anunciado su objetivo, los agentes ajustan sus expectativas. Si la tasa de inflación esperada coincide con la actual, la política monetaria no tendrá efectos reales. La desinflación se efectúa en un único periodo.

Cuando el banco central no es creíble, las expectativas no coinciden con los precios actuales, y la política monetaria tendrá consecuencias reales. En este caso particular, el precio que tendría que pagar la economía podría ser un aumento de la tasa de paro. En la práctica, por suerte, los agentes seguramente se darían cuenta de que los precios se acercan a los que anunció el banco central, y que en algún momento tendrían que reajustar sus expectativas.

\section{Conclusiones}

En este artículo planteamos un ejercicio que permite profundizar en el tema de la ejecución de la política monetaria en un modelo con expectativas. El ejercicio permite ver que el comportamiento de la economía depende del proceso de formación de expectativas. La ventaja de analizar el comportamiento de un modelo dinámico con la ayuda del ordenador es que se puede elaborar a la vez la solución numérica y gráfica. Es mucho más intuitivo ilustrar las soluciones gráficamente que en una tabla, como está hecho en el libro de Blanchard (2006), por ejemplo. Además es posible variar los parámetros de una manera muy flexible y comparar rápidamente distintas situaciones. La elaboración de un informe debería proporcionar tiempo de reflexión donde se puede adquirir el objetivo: entender las diferencias en el funcionamiento de la economía bajo diferentes supuestos de formación de expectativas, asociar el modelo con expectativas adaptativas al modelo keynesiano con precios rígidos, y el modelo con expectativas racionales y el banco central creíble con el modelo clásico y precios totalmente flexibles.

El ejercicio propuesto va más allá de una simple solución de un problema dado. Obliga al alumno a actuar como si fuera el director del banco central y observar enseguida las implicaciones que tendrían sus decisiones sobre una economía. Le facilitamos al alumno una herramienta de experimentación al estilo de un laboratorio. Un laboratorio para probar los efectos de distintas decisiones y objetivos; un laboratorio donde resulta muy fácil y barato diseñar nuevas estrategias y comprobar sus efectos.

Como complemento del ejercicio se pueden calibrar primero los parámetros $\alpha, \beta, \mathrm{u}_{N}$ y $\bar{\gamma}_{Y_{N}}$ utilizando los datos de algún país para el periodo del tiempo concreto, en este caso España entre 1962-2008. 


\section{Bibliografía}

Blanchard, Olivier (2000). Macroeconomía. Madrid: Pearson Educación, 2006. Trad. de Esther Rabasco Espáriz.

Novales, Alfonso y Sebastián, Carlos (1999). Análisis Macroeconómico I y II. Madrid: Marcial Pons, Ediciones Jurídicas y sociales.

\section{Anexo: Enunciado}

Se recomienda utilizar el programa Excel para la elaboración del trabajo.

Supón que la evolución de una economía se describe a través del siguiente sistema de ecuaciones

$$
\begin{aligned}
u_{t}-u_{t-1} & =-\frac{2}{7}\left(\gamma_{Y_{t}}-4,5\right), & & \text { (Ley de Okun) } \\
\pi_{t}-\pi_{t}^{e} & =-\frac{1}{7}\left(u_{t}-10\right), & & \text { (Curva de Phillips) } \\
\gamma_{Y_{t}} & =\gamma_{M_{t}}-\pi_{t}, & & \text { (Demanda agregada) } \\
\pi_{t+1}^{e} & =\theta \pi_{t}+(1-\theta) \pi_{t}^{e}, 0<\theta \leq 1, & & \text { (Expectativas adaptativas) }
\end{aligned}
$$

donde $u_{t}$ es la tasa de paro en el período $t, \gamma_{Y_{t}}$ es la tasa de crecimiento del output entre $t$ y $t-1, \pi_{t}$ y $\pi_{t}^{e}$ son las tasas de inflación actual y esperada, respectivamente, $\gamma_{M t}$ es la tasa de crecimiento de la oferta monetaria (nominal) entre ty $t-1$.

Supón que inicialmente la economía se encuentra en equilibrio y la oferta de dinero crece a una tasa de $19,5 \%$. Nota: Para solucionar el modelo en Excel hay que obtener primero una ecuación tipo

$$
\gamma_{Y_{t}}=f\left(\gamma_{M t}, u_{t-1}, \pi_{t}^{e}\right) .
$$

Esto se consigue sustituyendo la ley de Okun a la curva de Phillips y todo ello a la demanda agregada, y luego aplicar las expectativas.

Considera las expectativas totalmente adaptativas, $\theta=1$.

a) Calcula la tasa de inflación inicial.

El banco central anuncia el objetivo: reducir la tasa de inflación a $5 \%$. b) Aplica una política monetaria que reduciría la tasa de inflación al nivel deseado, aplicando la misma tasa de crecimiento del dinero en cada período. ¿Cómo variarán la tasa de crecimiento del output, la tasa de paro y la inflación en el tiempo? Apoya tus conclusiones con un diagrama de fase en el plano $(u, \pi)$ y un gráfico para la evolución de la tasa de crecimiento, tasa de paro y tasa de inflación.

c) Aplica ahora una política monetaria que reducirá la inflación poco a poco, aplicando una reducción de la tasa de crecimiento de la oferta monetaria de $5 \%$ cada 5 años, hasta llegar al nivel de inflación de $5 \%$. ¿Cómo variarán la tasa de crecimiento del output, la tasa de paro y la inflación en el tiempo? Apoya tus conclusiones con un diagrama de fase en el plano $(u, \pi)$ y un gráfico para la evolución de la tasa de crecimiento, tasa de paro y tasa de inflación.

d) Compara los niveles de paro y tasa de crecimiento del output en el caso b) y c) (un gráfico para la evolución del paro, otro para la tasa de crecimiento de la economía). Comenta.

Considera que la desinflación es perfectamente creíble y la inflación esperada es de 5\%. Eso implica que cambiamos la ecuación de expectativas

$\pi_{t}^{e}=E_{t-1}\left(\pi_{t}\right)$.

e) ¿Qué pasa en la economía? Explica el papel de las expectativas y la importancia de la credibilidad del Banco central.

Considera que la gente no cree que el banco central será capaz de reducir la inflación tanto y la inflación esperada es de $10 \%$ (expectativas racionales pero con otro nivel de esperanza sobre la tasa de inflación).

f) ¿Qué pasa en la economía? Explica. Utiliza gráficos que te puedan ayudar a sacar las conclusiones. 